\title{
Loss of discordant cells during micro-mass differentiation of embryonic stem cells into the chondrocyte lineage
}

\begin{abstract}
A Yamashita ${ }^{1}$, R Krawetz ${ }^{1}$ and DE Rancourt ${ }^{*, 1}$
Embryonic stem cells (ESCs) have attracted particular interest in regenerative medicine because of their unlimited self-renewal and multipotentiality for differentiation. Spontaneous differentiated ESCs display heterogeneous multipotent cell populations and generate teratomas in vivo, with process by which ESCs differentiate into specific lineages remaining unclear. In this study, we focused on the in vitro chondrocyte differentiation of ESCs through micro-mass without using an embryoid body (EB) step and observed the unique characteristics of cartilage formation coupled with endochondral ossification in vivo. This approach resulted in an aggressive loss of discordant cells by apoptosis, which was accompanied by significant changes in gene expression during the course of ESC differentiation into chondrocytes. Unlike EB formation where discordant cells remain trapped within aggregates, micro-mass permits cells to die, leave the group and/or form a new group in response to changes in gene expression. Our observations suggest that the cell death that accompanies ESC micro-mass differentiation helps purify a terminally differentiated cell population and selects for targeted end points within a suitable microenvironment.
\end{abstract}

Cell Death and Differentiation (2009) 16, 278-286; doi:10.1038/cdd.2008.149; published online 17 October 2008

Embryonic stem cells (ESCs) display self-renewal and pluripotency. ${ }^{1,2}$ In essence, ESCs offer a potentially unlimited supply of cells that may be driven down specific lineages to give rise to all cell types in the body. ${ }^{3,4}$ One of the challenges is to understand, control and develop an efficient and stable culture milieu for directing differentiation into lineage-specific progenitors or terminally differentiated cells. ${ }^{5}$ As ESCs are derived from the inner cell mass, their differentiation can be used to recapitulate early embryonic development. To this end, two methods have been used in an attempt to stimulate the differentiation of ESCs into particular lineages. In one method, ESCs are allowed to form embryoid body (EB), mimicking the structure of the early embryo and creating suitable conditions for stochastic differentiation into mesoderm, ectoderm and endoderm. ${ }^{3,4,6}$ Here, the addition of growth factors helps to influence differentiation toward particular lineages. In another, more recent method, ESCs have been differentiated directly on culture dishes without EB formation. ${ }^{7}$ Recently, for example, it has been reported that such direct differentiation of human and mouse ESCs results in a significantly greater number of osteogenic cells. ${ }^{8,9}$

It has been reported that a supportive three-dimensional (3D) environment that more closely mimics chondrogenesis in vivo will induce distinct cellular behavior not present in monolayer culture. ${ }^{10,11} 3 \mathrm{D}$ culture systems in the form of pellet or micromass have been frequently used for efficient chondrogenesis of mesenchymal stem cells (MSCs) or chondro-progenitor cells. ${ }^{12}$
Several groups have described cell-intrinsic selection processes that take place during terminal differentiation. ${ }^{13-15}$ In these studies, gene silencing allows for selection against discordant cell types and improves the purity of the desired target lineage. However, these studies also demonstrate that increased rates of apoptosis are not essential for this mechanism.

Here, we describe the chondrocyte differentiation of mouse ESCs using micro-mass culture in the absence of EB formation. Using this method, lineage restriction as demarcated by changes in lineage-specific gene expression is accompanied by massive apoptosis and the departure live cells, which form aggregates in suspension and subsequently adhere to form cartilage masses in vitro. In contrast to other methods that require 15 or 30 days to form chondrocytes in the absence or presence of EB formation, ${ }^{16,17}$ using a micromass approach we have observed relatively pure masses of cartilage formed in 6 days.

\section{Results}

ESC morphology changes during micro-mass differentiation. Mouse ESCs were differentiated using a micro-mass method without EB formation. Using protocols similar to MSCs and chondro-progenitor cells, ESCs were cultured at high density $\left(1.0 \times 10^{5}\right.$ cells per $10 \mu \mathrm{l}$ drop) for $2 \mathrm{~h}$. To examine cell growth and differentiation, we tested three media

\footnotetext{
${ }^{1}$ Department of Biochemistry \& Molecular Biology, University of Calgary, 3330 Hospital Drive NW, Calgary, Alberta, Canada

*Corresponding author: DE Rancourt, Department of Biochemistry \& Molecular Biology, University of Calgary, 320, 3330 Hospital Drive NW, Calgary, Alberta, Canada T2N 4N1. Tel: + 1403220 2888; Fax: + 1403270 0834; E-mail: rancourt@ ucalgary.ca

Keywords: embryonic stem cell; micro-mass; chondrogenesis; apoptosis; gene regulation

Abbreviations: ESCs, embryonic stem cells; EB, embryoid body; MSCs, mesenchymal stem cells; ITS, insulin, transferrin and selenium; CS, chondrogenic supplement; 3D, three dimensional; GAG, glycosaminoglycan; COL, collagen; PI, propidium iodide; H\&E, hematoxylin and eosin; NEAA, non-essential amino acids; Pen/Strep, penicillin and streptomycin; $\beta \mathrm{ME}$, 2-mercaptoethanol

Received 27.6.08; revised 09.9.08; accepted 09.9.08; Edited by RA Knight; published online 17.10.08
} 
formulations: ITS-CS (insulin, transferrin and seleniumchondrogenic supplement), ITS-basic and FBS-CS. In the ITS-CS and ITS-basic treatment groups, cells aggregated spontaneously and lifted off the culture surface after 4 days (Figure $1 \mathrm{Ab}$ and $\mathrm{Ae}$ ). In FBS-CS conditions, cells remained adhered and expanded peripherally to the central micro-mass (Figure 1Ah). The floating aggregates observed under ITS-CS and ITS-basic conditions reattached within $24 \mathrm{~h}$ (day 5) and from this point onwards striking morphological differences between ITS-CS and ITS-basic were observed (Figure 1Ac and $\mathrm{Ae}$ ). Under ITS-CS treatment, the aggregates retained a uniform and distinct margin (Figure $1 \mathrm{Al}$ and $\mathrm{Ba}$ ), as compared to ITS-basic aggregates that displayed irregular and undefined margins (Figure $1 \mathrm{Ao}$ and $\mathrm{Bb}$ ).

We observed clear differences in aggregate size and growth during the differentiation period and proceeded to examine this phenomenon. The average size of ESCs on day 0 was $16 \mu \mathrm{m}$, and decreased immediately after induction (14 $\mu \mathrm{m}$ on day 2) (Figure 1C). Moreover, there were two more distinct decreases in diameter, which occurred on day 3
A

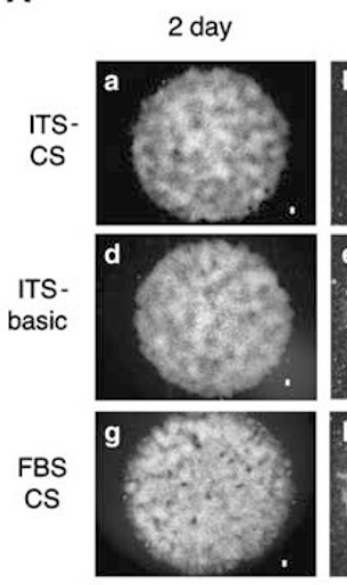

2 day

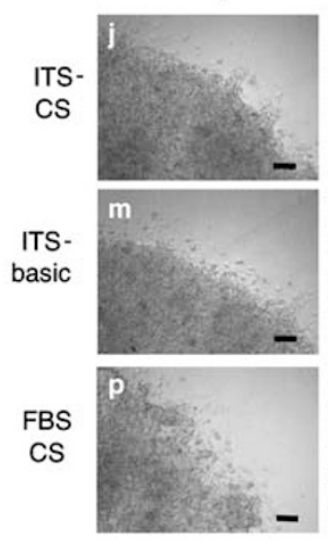

B
ITS-CS

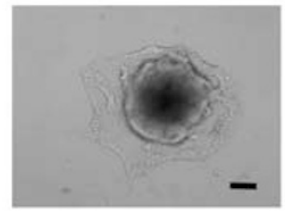

4 day

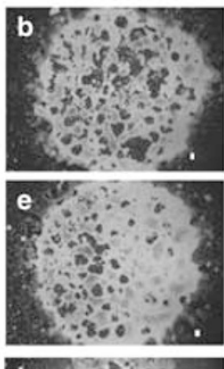

6 day

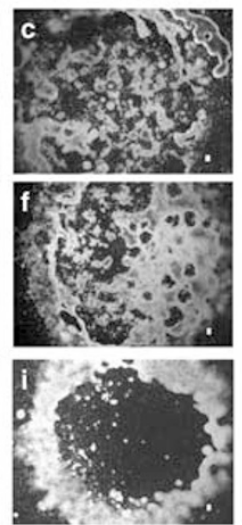

6 day
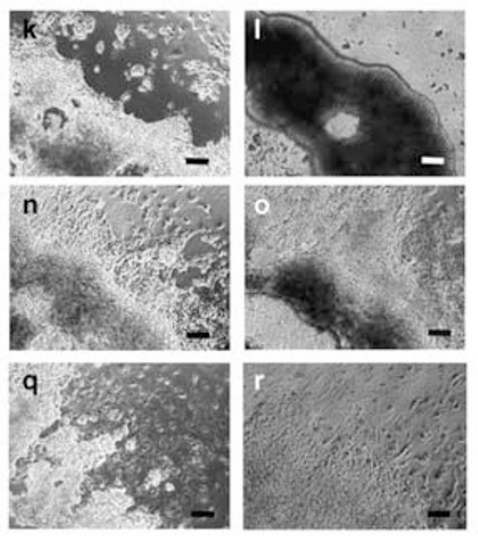

ITS-basic

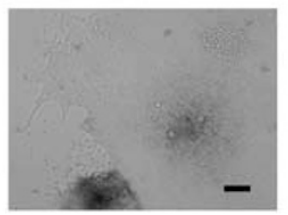

C

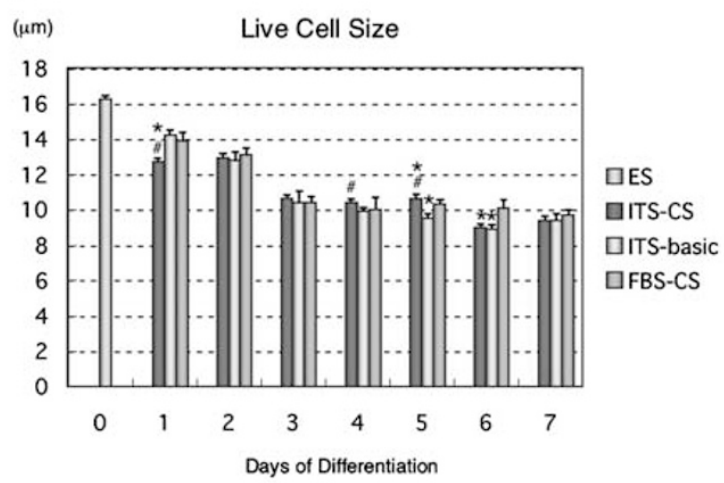

D

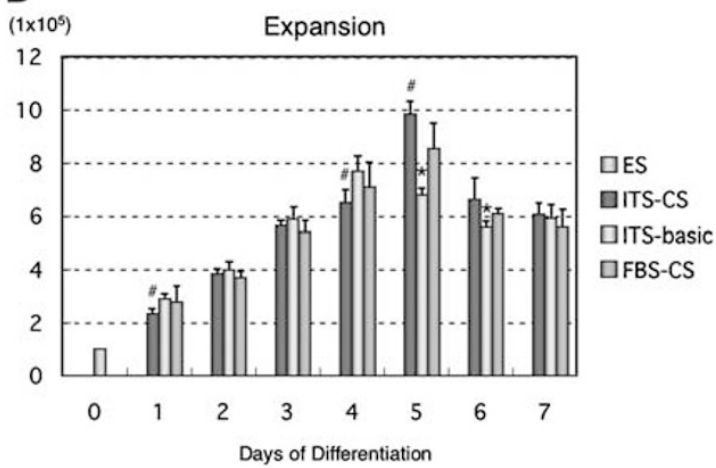

E

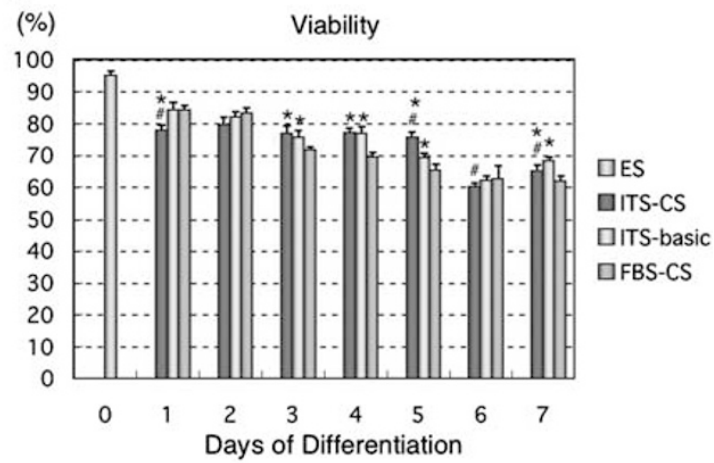

Figure 1 Embryonic stem cell morphology during micro-mass culture. (A and $\mathbf{B}$ ) Differentiation of ESCs using micro-mass culturing techniques resulted in dramatic changes in morphology between the three media conditions tested: ITS-CS (Aa-C and j-I), ITS-basic (Ad-f and $m-0$ ) and FBS-CS (Ag-i and p-r). On day 4 of differentiation, ITS-CS (Ab) and ITS-basic (Ae) detached from the substrate only to re-attach by day 6 ( $\mathbf{A} \mathbf{c}$ and $\mathbf{A}$, respectively). FBS-CS cultured cells did not detach (Ah), but grew off the central cell mass (Ai). Increased magnification at the end of the micro-mass ( $\mathbf{A} \mathbf{j}-\mathrm{r})$ shows the difference between ITS-CS (Al) and ITS-basic (Ao) after re-attachment, which is easily detected at lower magnifications ( $\mathrm{Ba}$ and $\mathrm{Bb}$, respectively). (C-E) The size of individual cells within the micro-mass pellets was assayed from day 0 to 7 of differentiation (C), as were culture expansion rates (D) and cell viability (E). Data are expressed as means \pm S.D. $(n=3)$ per lane. "Significant difference from ITS-basic, $P<0.05$ with Student's $t$-test. *'Significant difference from FBS-CS, $P<0.05$ with Student's $t$-test. Scale bars represent $100 \mu \mathrm{m}$ 
$(10 \mu \mathrm{m})$ and day $6(9 \mu \mathrm{m})$. Once the cells reached $6 \mu \mathrm{m}$, there was no significant increase or decrease in cell diameter (Figure 1C).

Viability of differentiated ESCs. We performed daily cell counts and viability assays on micro-mass cultures to track proliferation rates (Figure $1 \mathrm{D}$ and E). Differentiated ESC growth rates increased gradually and peaked at day 5 in ITS-CS and FBS-CS conditions (Figure 1D). The growth rate of ITS-basic was slightly faster and peaked at day 4 of differentiation (Figure 1D). We then assayed the viability of the cells during induction and found that viability decreased immediately at the beginning of differentiation (Figure 1E). ITS-CS cultured cells retained a $75 \%$ viable cell density until day 5 , after which the viability level decreased. In FBS-CS conditions, cell viability decreased gradually. It should also be noted that cell proliferation and viability rates between ITS-CS and ITS-basic treatment groups were significantly different (Figure 1D and E).

Modulation of chondrogenesis in vitro. We further investigated chondrocyte differentiation within ESC micromass culture in vitro, by analyzing the accumulation of glycosaminoglycans (GAGs), and chondrocyte-specific mRNA and protein levels (Figure 2). GAG levels were upregulated during the course of differentiation in ITS-CS conditions (Figure 2A). When normalized to DNA content, the corrected GAG/DNA analysis showed continuously low levels of GAGs in ITS-basic and FBS-CS treatment groups, as compared to ITS-CS conditions that displayed a significant increase in GAG/DNA levels (Figure 2A). Alcian Blue, which binds to proteoglycans, was used to visually confirm the GAG quantitative analysis. In ITS-CS conditions, Alican Blue staining was present at day 6 and was found in a cartilage nodule-like pattern (Figure $2 \mathrm{Ba}$ ) and was also present at the edge of the micro-mass (Figure 2Bb). This staining was retained when the aggregates lifted off the substrate and also during the re-attachment phase (Figure 2Bc and d).

In addition to GAG accumulation, we also examined the calcium content of the micro-mass cultures (Figure $2 \mathrm{C}$ ). Increased calcium levels are normally associated with bone, however, cartilage does accumulate high levels of calcium, especially when undergoing endochondral ossification. ${ }^{18,19}$ Calcium levels in all treatment groups increased during differentiation, with higher calcium/DNA levels observed in the ITS-CS conditions (Figure 2C). Although GAG content is indicative of cartilage formation, we also chose to directly examine specific molecular markers known to be expressed during chondrocyte differentiation.

Semiquantitative RT-PCR demonstrated that micro-mass ESCs expressed Aggrecan and collagen (COL) 2 mRNA in all culture conditions (Figure 2D), both of which are early markers of chondrocyte differentiation. SOX9 mRNA, a transcription factor associated with chondrocyte differentiation was expressed in ITS-CS conditions at day 6 (Figure 2D). Interestingly, COL $10 \mathrm{mRNA}$, which is a late marker involved with hypertropic maturation, was also expressed strongly in ITS-CS conditions at day 6 (Figure 2D). Immunofluorescence was carried out in ITS-CS cells on day 7 and showed positive staining for Aggrecan (Figure 2Ea) and COL 2 (Figure 2Ed). COL 10 deposition was also observed, however, the staining was noticeably weaker (Figure 2Eg).

Modulation of chondrogenesis in vivo. We investigated the ability of micro-mass culture to differentiate into chondrocytes in vivo by injecting dissociated day 4 micromasses into SCID mice. These in vitro differentiated cells continued to proliferate and differentiate in vivo, and after 4 weeks, the extracted tissue retained defined margins (Figure 3A). The different culture conditions produced tissues that ranged in weight and area, $16 \mathrm{mg} / 0.015 \mathrm{~cm}^{2}$ (ITS-CS; Figure 3Aa), $606 \mathrm{mg} / 1.34 \mathrm{~cm}^{2}$ (ITS-basic; Figure $3 \mathrm{Ab}$ ) and $200 \mathrm{mg} / 0.62 \mathrm{~cm}^{2}$ (FBS-CS; Figure 3Ac).

On the basis of histology of these tissues, ESCs treated with ITS-CS generated cartilage and connective tissues (Figure $3 \mathrm{Ba}$ and $\mathrm{Bb}$ ), and the cartilage became dark blue after Alcian Blue staining (Figure $3 \mathrm{Bc}$ and $\mathrm{Bd}$ ). These differentiated cells were therefore also abundant with matrix consisting of GAG. On closer examination, we also observed bone and blood vessels in these extracted tissues (Figure $3 \mathrm{C}$ ). The bone that was generated around the cartilage stained positive for Alizarin Red S (Figure $3 \mathrm{Cd}$ and $\mathrm{Ce}$ ), and appeared to be the result of endochondral ossification during the 4-week in vivo differentiation period.

In contrast to the ITS-CS conditions, we observed a loss of tissue specificity in vivo within ITS-basic and FBS-CS conditions where cartilage, bone, fat, blood and muscle were produced (Figure 3D and E). These two media conditions also formed teratomas in vivo where the ITS-CS treatment group did not.

Micro-mass specification toward chondrogenesis. Based on these results, we sought to profile the expression of lineage markers during differentiation under all three media conditions, using semiquantitative RT-PCR (Figure 4A). Using ITS-CS conditions, Nestin (ectoderm), AFP (endoderm) and Stella (reproduction) were downregulated and Brachyury (mesoderm) was upregulated at day 6 . In addition, Osteocalcin (bone), aP2 (fat) and $\alpha$-actin (muscle) were downregulated and Flk1 (mesoderm, blood vessel) was upregulated (Figure 4A). Interestingly, ITS-basic strongly expressed Osteocalcin on day 6 and only displayed weak bands for AFP, aP2 and $\alpha$-actin, whereas FBS-CS expressed all targets tested (Figure 4A).

Aggressive apoptotic cell loss during chondrocyte differentiation. As ITS-CS cultures lost $25 \%$ of viable cells in vitro and also produced significantly smaller tissue masses in vivo, we sought to determine whether such aggressive cell loss might be explained by apoptosis (Figure 4B and C). On day 4 of in vitro differentiation, we observed extensive TUNEL-positive cells in the margin of the micro-masses (Figure 4Ba). By day 4, many aggregates detached and we observed TUNEL-positive cells remaining on the culture dish (Figure 4Bc). Early apoptotic cells, which were Annexin V positive and propidium iodide $(\mathrm{PI})$ negative, accounted for $15 \%$ of the total population on days 1 and 2 , increasing to $25-35 \%$ on days $3-5$ (Figure $4 \mathrm{C}$ ). 
A

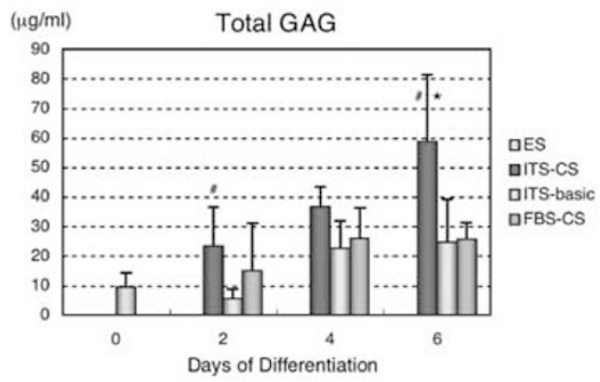

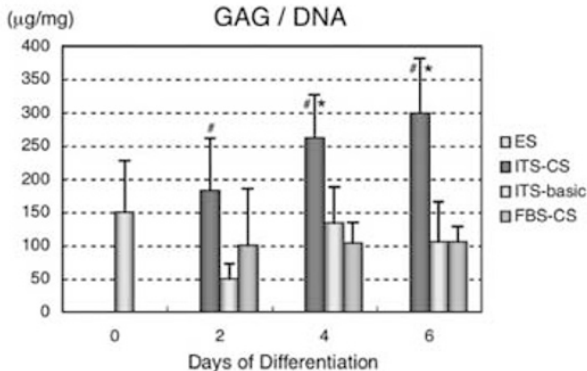

B
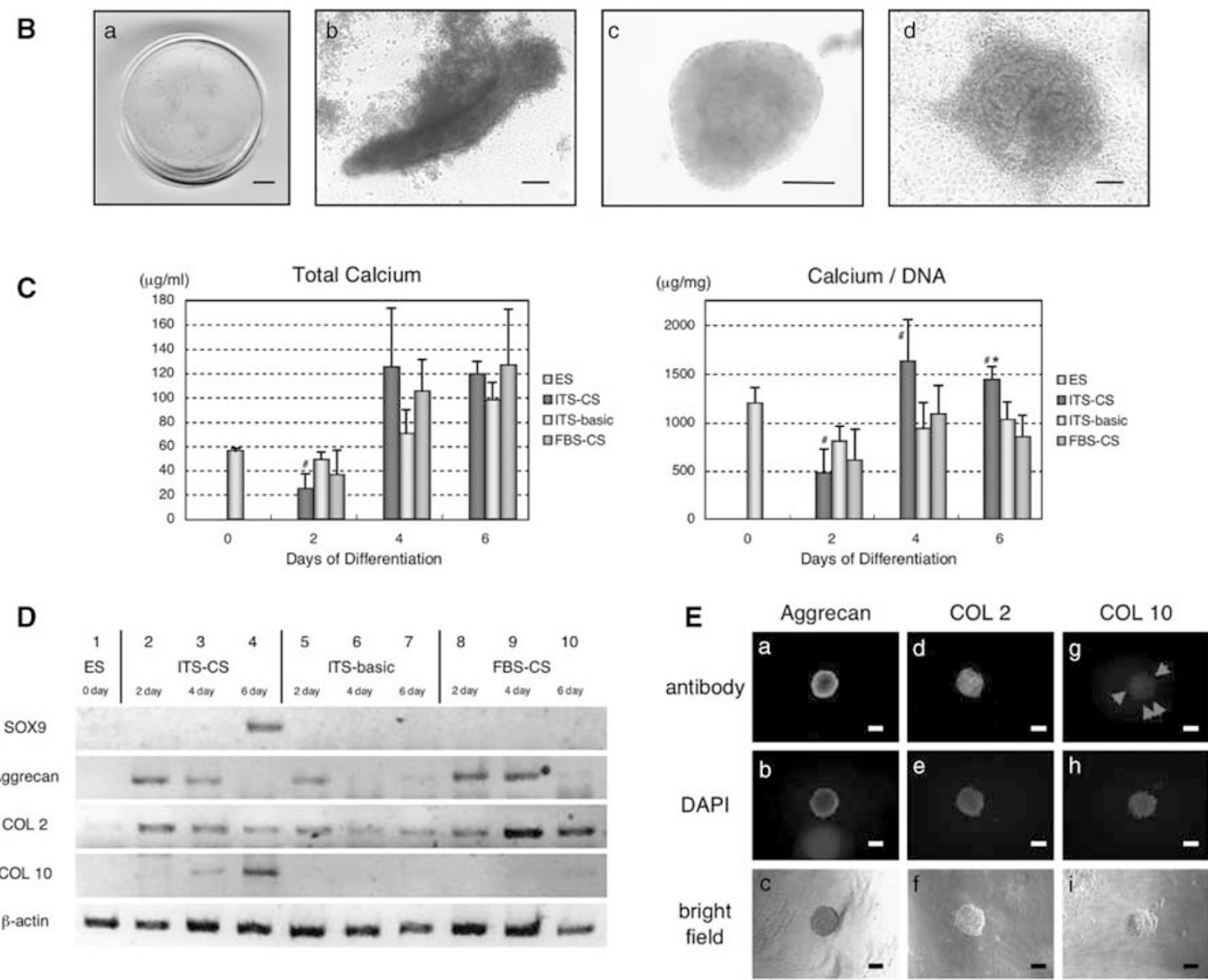

Figure 2 Chondrocyte characteristics during micro-mass in vitro differentiation. (A) GAG content per micro-mass spot was determined (A, left). GAG content was also normalized against DNA content to determine GAG synthesis per cell (A, right). Data are expressed as mean \pm S.D. $(n=4)$ per well. " Significant difference from ITS-basic, $P<0.05$ with Student's $t$-test. *Significant difference from FBS-CS, $P<0.05$ with Student's $t$-test. (B) Using Alcian Blue staining, cartilage-like nodules with dark blue appearance were observed in culture dish on day 6 of differentiation (Ba). Alcian Blue staining was observed on the edge of micro-mass (Bb). Floating aggregates also stained blue (Bc). Once these aggregates re-attached, Alcian Blue-positive cells were seen throughout the micro-mass (Bd). Scale bars represent $500 \mu \mathrm{m}$ for (Ba), and $100 \mu \mathrm{m}$ for $(\mathrm{Bb}-\mathrm{d})$. (C) Calcium content per micro-mass spot was determined $(\mathbf{C a})$. Calcium content was also normalized against DNA content to determine calcium synthesis per cell (Cb). Data are expressed as mean \pm S.D. $(n=4)$ per well. " Significant difference from ITS-basic, $P<0.05$ with Student's $t$-test. *Significant difference from FBS-CS, $P<0.05$ with Student's t-test. (D) The expression of chondrocyte-related mRNAs (SOX9, Aggrecan, COL 2, COL 10) was analyzed using semiquantitative RT-PCR at 2, 4 and 6 days. $\beta$-actin was used as a loading control. (E) The expression of chondrocyte-related proteins in ITS-CS conditions (Aggrecan, COL 2, COL 10) was analyzed by immunofluorescence on day 7 . Scale bars represent $100 \mu \mathrm{m}$

\section{Discussion}

The process of chondrogenic differentiation is influenced by a number of growth factors, including TGF- $\beta$ and/or BMPs, and is measured by the sequential expression of specific matrix components. ${ }^{20}$ Earlier, we and others have developed methods to differentiate ESCs into chondrocytes by exposing EBs to TGF- $\beta$ and/or BMP-2. ${ }^{16,19,20-24}$ Using EB differentiation, it takes approximately 30 days to express markers of chondrocyte differentiation, including Aggrecan and $\mathrm{COL} 2,{ }^{16}$ as compared to other methods that remove the EB step that require only 15 days in culture. ${ }^{17}$ It is important to note, 

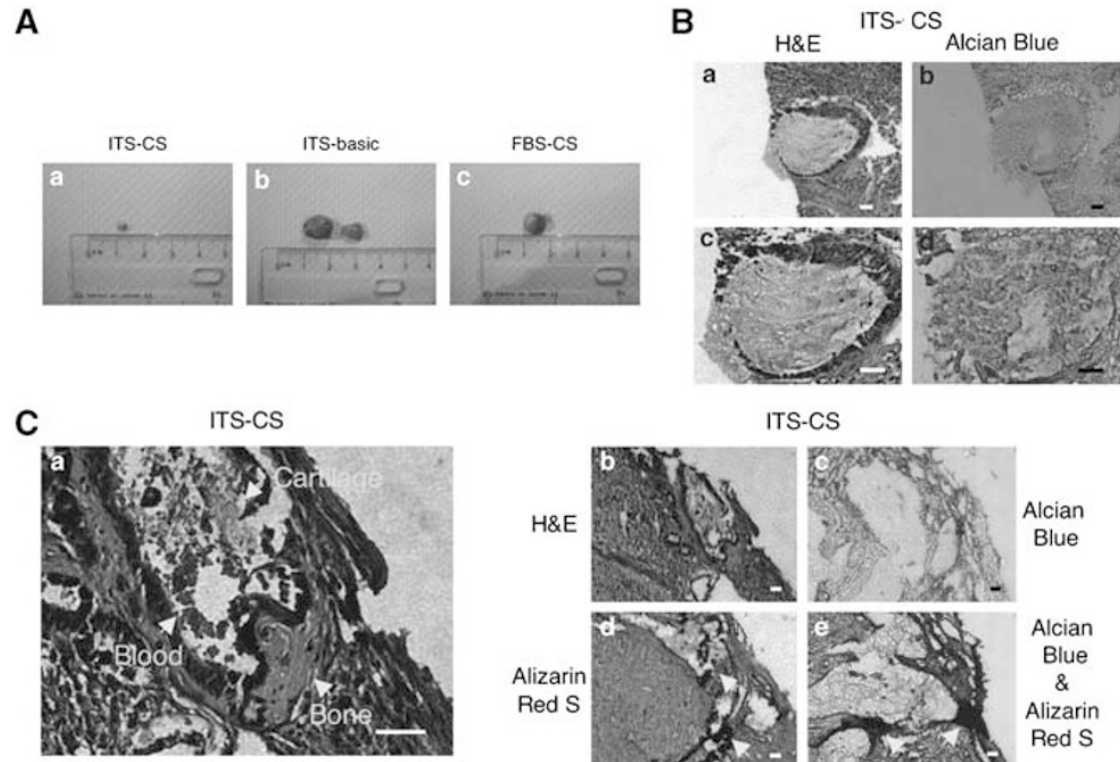

D
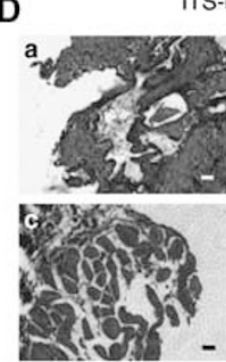

S-basic
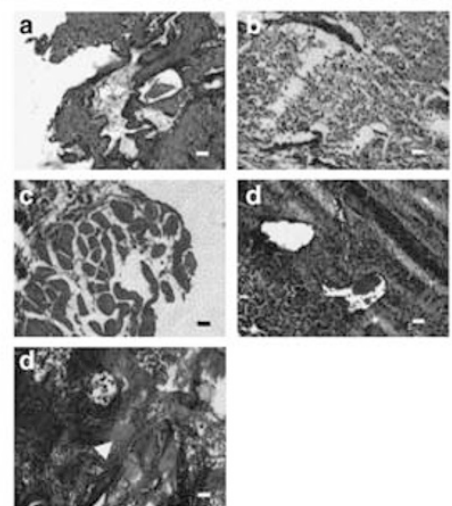

ITS-CS

FBS.CS
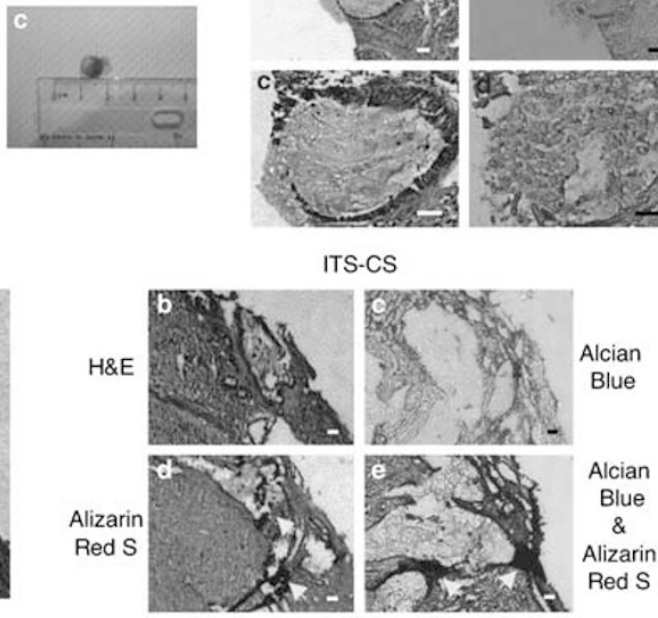

$\mathrm{E}$

FBS-CS
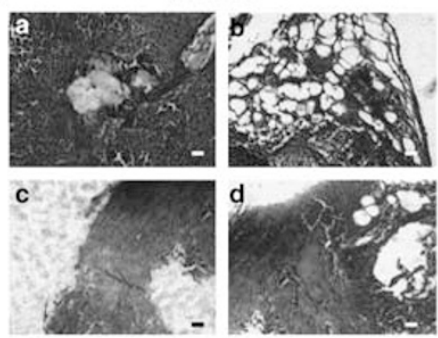

Figure 3 Differentiation of micro-mass cultures in vivo. ESCs differentiated in ITS-CS, ITS-basic or FBS-CS were collected from micro-drops on day 4 of differentiation, whereupon $4.5 \times 10^{5}$ cells were injected i.m. into SCID mice. At 4 weeks after injection, the resulting tissue was excised, measured (A), and histologically stained (B-E). ITSbasic and FBS-CS conditions resulted in large tissue masses (Ab and $c$ ), whereas the size of tissue masses was relatively restricted under ITS-CS conditions. Under ITS-CS conditions, cartilage and connective tissue formed in vivo (Ba-d). ITS-CS-derived cartilage stained positive for Alcian Blue (Bc and d). H\&E staining of tissue section viewed at a higher magnification showed that not only cartilage but also blood and bone are detected in ITS-CS (Ca). ITS-CS tissue sections stained with H\&E (Cb). Alcian Blue staining was positive $(\mathbf{C} \mathrm{c})$, demonstrating the presence of cartilage. Alizarin Red S staining of tissue section $(\mathbf{C d})$ showed presence of bone. Alcian Blue co-staining with Alizarin Red S shows interaction between bone and cartilage tissues (Ce). Under ITS-basic conditions, the teratomas contained cartilage (Da), bone (De), fat (Db), muscle (Dc) and blood vessels (Dd). Under FBS-CS conditions, the teratomas contained cartilage (Ea), fat (Eb), muscle (Ec) and blood vessel (Ed). Scale bars represent $50 \mu \mathrm{m}$

however, that the previous report describing ESC-derived chondrogenesis without EB formation does not provide any in vivo data that prove the generation of functional cartilage. $^{17}$

Two recent observations influenced our decision to explore ESC chondrocyte differentiation using micro-mass culture and avoiding the EB step. First, it was recently reported that ESCs have the capacity to differentiate spontaneously into osteoblasts without EB formation and offering the advantage of streamlining differentiation., 8 Second, 3D high-density micro-mass culture also allows immediate cell condensation to form cartilage-like nodule ${ }^{25}$ and is commonly used in the chondrocyte differentiation of MSCs and chondrogenic progenitors. ${ }^{10-12}$

To examine the capacity of ESCs to differentiate toward chondrogenesis, we explored the use of the CSs
TGF- $\beta$ and BMP-2 in minimal defined media supplemented with ITS, ingredients commonly found in serum replacement formulations. Although we attempted to completely remove serum from the ITS formulations (i.e. ITS-basic and ITS-CS), ${ }^{20,23}$ we observed that $1 \%$ FBS was necessary to facilitate ESC attachment in micro-mass spots. With the significant cell death and/or dispersal, which occurred under ITS conditions, we have not explored whether 1\% FBS can be removed once micro-masses formed. Indeed, this cell death/dispersal was not observed in FBS-CS conditions, where cultures are supplemented with normal levels of FBS.

Under these culture conditions, micro-mass ESCs expanded and changed morphology. Under both ITS-CS and ITS-basic conditions, a significant number of cells 
A

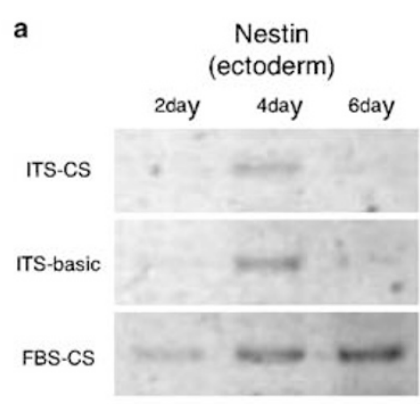

d

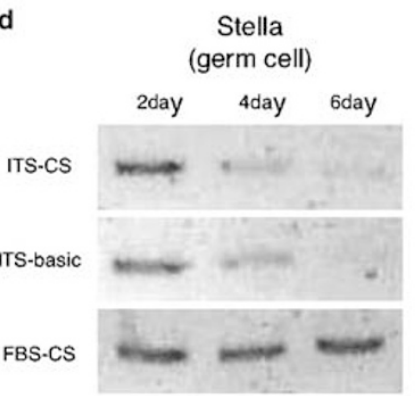

g

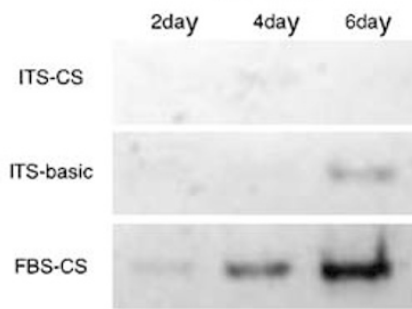

b

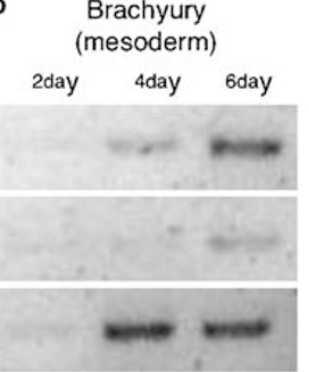

e

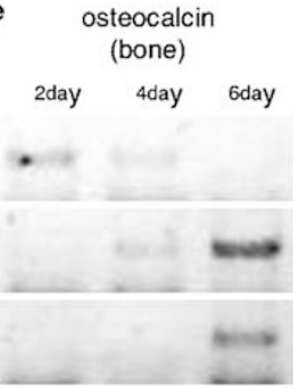

h

Flk 1 (blood vessel)

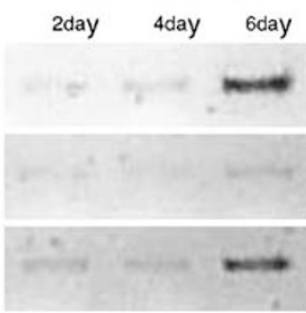

c

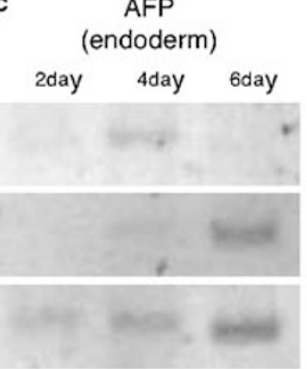

f

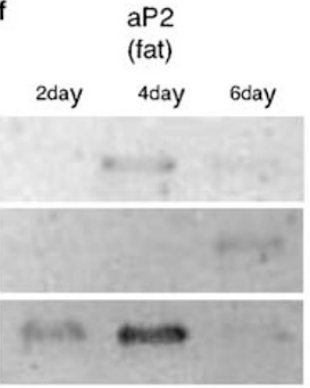

$\beta$-actin

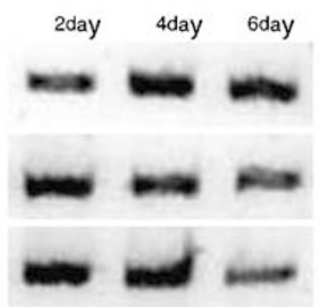

B
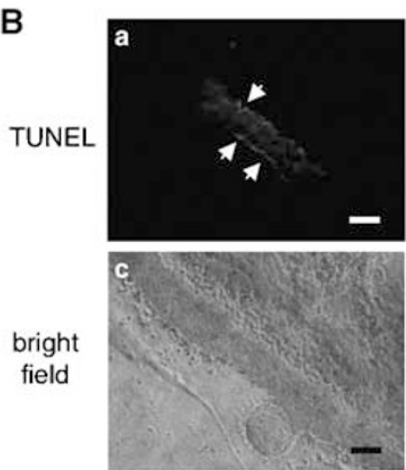
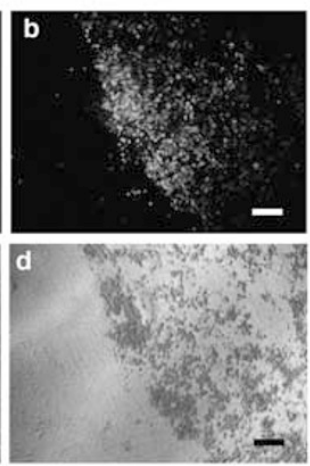

C

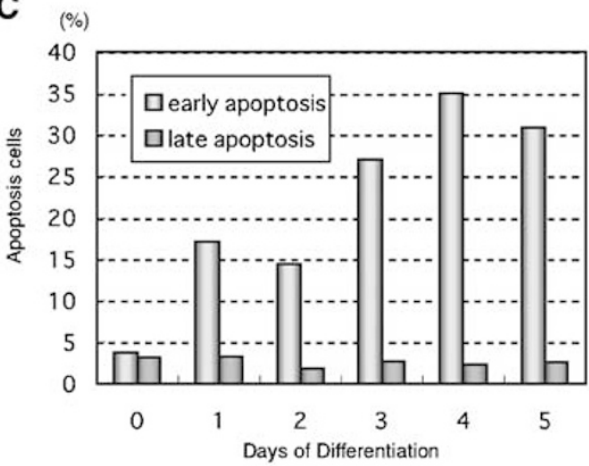

Figure 4 Apoptosis during chondrogenic lineage restriction. (A) Semiquantitative RT-PCR was used to detect the expression of Nestin (ectoderm, Aa), Brachyury (mesoderm, Ab), AFP (endoderm, Ac), Stella (germ cell, Ad), Osteocalcin (bone, Ae), aP2 (fat, Af), $\alpha$-actin (muscle, Ag), Flk1 (blood vessel, Ah), and $\beta$-actin (Ai) at 2, 4 and 6 days. (B) Using TUNEL staining, apoptotic cells were observed in the margin of ITS-CS-treated cells (Ba, arrows), the corresponding brightfield channel demonstrates cell position within the micro-mass $(\mathrm{Bb})$. After micro-mass aggregates detached from the substrate, TUNEL-positive cells were observed on the culture dish (Bc, brightfield $\mathbf{B d}$ ). Scale bars represent $100 \mu \mathrm{m}$. (C) Annexin V-FITC/PI labeling was used to detect and quantify apoptotic cells in ITS-CS conditions over differentiation. In total, 10000 events were registered per sample

detached from the substrate between days 2 and 4 and spontaneously formed aggregates, which grew in suspension within micro-drops. In contrast, FBS-CS-treated cells remained attached and expanded peripheral to the spotted micro-mass spot. Interestingly, the ITS aggregates returned to the substrate by day 5 . 
After reattachment of aggregates in ITS-CS and ITS-basic conditions, we observed striking morphological differences between CS and basic conditions (Figure 1B). Unlike ITS-basic aggregates that displayed a rather disorganized configuration, ITS-CS reattachments appeared highly organized, bearing strikingly sharp margins. In addition, only ITS-CS-treated cells expressed cartilage-specific extracellular matrix proteins and SOX9, a transcription factor expressed during chondrogenesis (Figure 2). During the process of differentiation in vitro, ITS-CS-treated cells differentiated into hypertrophic chondrocyte and mineralized. ${ }^{19,21}$ It is likely that the spontaneous aggregates that formed in ITS-CS developed a supportive 3D environment that more closely mimicked chondrogenesis in vivo. Injection of ITS-CS-treated cells into SCID mice resulted in small tissue masses comprised predominantly of cartilage (Figure $3 \mathrm{~A}$ and $\mathrm{B}$ ). In contrast, cells derived from ITS-basic and FBS-CS conditions resulted in teratomas (Figure $3 \mathrm{D}$ and E). Although we also observed small amounts of blood and bone tissue in ITS-CSderived tissue (Figure $3 C$ ), we attribute this to endochondral ossification, a natural process whereby cartilage is replaced by bone tissue following vascular invasion..$^{20,24}$ This observation is not unprecedented, and may even occur during in vitro differentiation. ${ }^{16}$ This latter observation may help to explain why we detect some mineralization in ITS-CS cultures (Figure 2C), despite the clear absence of osteoblast marker (i.e. Osteocalcin) expression (Figure 4Ae).

Only under ITS-CS conditions, did we see a progressive loss of ectodermal, endodermal and germ-cell marker expression and a concomitant increase in mesodermal marker expression. By day 6 of differentiation, evidence of bone, fat and skeletal muscle was non-existent. However, evidence of blood vessel differentiation persisted, as detected by the expression of Flk1. This result may help to explain the notion of endochondral ossification, which we observed in vivo.

In MSC differentiation, it has been suggested that the downregulation of unrelated phenotypic markers and cellular properties results from negative selection that is manifested by a combination of apoptosis and/or transcriptional regulation during the specific-lineage differentiation. ${ }^{14}$ It stands to reason that a similar process may operate during ESC differentiation. We observed a massive decrease of cell viability following induction, which continued until day 6 (Figure 1E). Annexin apoptosis detection by FACScan analysis demonstrated that early apoptotic cells increased within the population immediately after induction and again at day 3 (Figure 4C). More aggressive cell loss appeared to start at day 3 , which was accompanied by a decrease in average diameter of live cells in ITS-CS conditions (Figure 1C). A decrease in cell diameter is an early hallmark of apoptosis. ${ }^{26}$ This, coupled with the TUNEL analysis (Figure 4B), implies an increase in cell death during the onset of the differentiation protocol. We suspect that if we allowed the experiment to proceed for a longer period of time, we would observe an increase in cell diameter in the ITS-CS treatment group indicative of chondrocyte differentiation. ${ }^{27}$ The apoptotic cells appeared in the edge of micro-masses and remained on the culture surface after aggregates detached (Figure 4B). These results demonstrated that aggressive cell loss by apoptosis occurred during chondrogenic differentiation. We suggest that differentiation may not only require the increase of phenotypespecific cells, as manifested by the expression of lineagespecific markers, but also the decrease of discordant cells as mediated by apoptosis. ESCs might undergo a lineagespecific differentiation resulting in apoptosis of discordant cells along with changes in cellular morphology and microenvironment (Figure 5). This ability of discordant cells to die or depart may help to explain the accelerated differentiation/ development occurring within micro-mass culture. For example, such behaviors may not be possible with cells trapped within EBs. Hence differentiation/development within EBs may be delayed through a process of cellular negotiation where differentiating cells more slowly find their way through compromise.

\section{Materials and Methods}

Mouse ESC culture. The D3 mouse ESC line ${ }^{28}$ was cultured on mitomycin Ctreated $\left(10 \mu \mathrm{g} / \mathrm{ml}, 37^{\circ} \mathrm{C}\right.$ for $2 \mathrm{~h}$; Sigma) mouse embryonic fibroblast feeder layers on gelatin-coated tissue culture dishes. ${ }^{29,30} \mathrm{ESC}$ medium consisted of high glucose DMEM (4.5 g glucose/l; Gibco) containing 15\% FBS (Gibco), 1\% non-essential amino acids (NEAA; Invitrogen), $50 \mathrm{U} / \mathrm{ml}$ penicillin and $50 \mu \mathrm{g} / \mathrm{ml}$ streptomycin (Pen/Strep; Invitrogen), $0.1 \mathrm{mM}$ 2-mercaptoethanol ( $\beta \mathrm{ME} ;$ Invitrogen) and $1000 \mathrm{U} / \mathrm{ml}$ leukemia inhibitory factor (Invitrogen), as described earlier. ${ }^{31}$

Micro-mass culture. Micro-mass culture was performed as described earlier. $^{12}$ Briefly, ESCs were dissociated using $0.1 \%$ trypsin-EDTA. The dissociated cells were cultured at a high density of $1.0 \times 10^{5}$ cells per $10 \mu \mathrm{l}$ spot for $2 \mathrm{~h}$. After incubation, medium was gently added to each dish so as to not dissociate the aggregates. For chondrocyte differentiation, we used three media

The selection and elimination process

in chondrocyte-lineage differentiation of ESCs

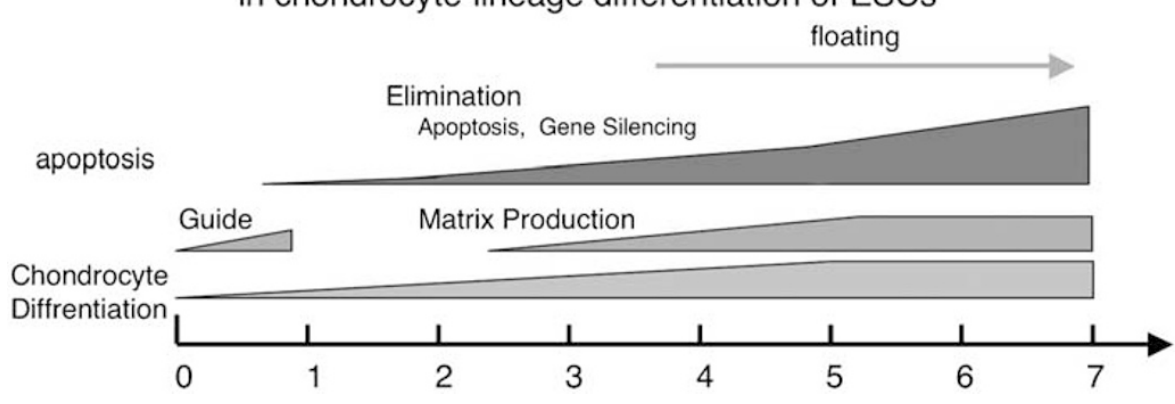

Figure 5 Cell selection and elimination during chondrogenic differentiation of ESCs in micro-mass. Guided differentiation of ESCs can be achieved, in part, due to the progressive loss of discordant cells coupled within induction into the chondrocyte lineage 
Table 1 Primer sequences and cycle number

\begin{tabular}{|c|c|c|c|}
\hline Genes & & Primer & Cycles \\
\hline sox9 & $\begin{array}{l}\text { Forward } \\
\text { Reverse }\end{array}$ & $\begin{array}{l}\text { 5'-AGCTCACCAGACCCTGAGAA-3' } \\
\text { 5'-GATTCTCCAATCGTCCTCCA-3' }\end{array}$ & 35 \\
\hline Aggrecan & $\begin{array}{l}\text { Forward } \\
\text { Reverse }\end{array}$ & $\begin{array}{l}\text { 5'-CAGGGTTCCCAGTGTTCAGT-3' } \\
\text { 5'-CTGCTCCCAGTCTCAACTCC-3' }\end{array}$ & 30 \\
\hline COL 2 & $\begin{array}{l}\text { Forward } \\
\text { Reverse }\end{array}$ & $\begin{array}{l}\text { 5'-CCGTCATCGAGTACCGATCA-3' } \\
\text { 5'-CAGGTCAGGTCAGCCATTCA-3' }\end{array}$ & 30 \\
\hline COL 10 & $\begin{array}{l}\text { Forward } \\
\text { Reverse }\end{array}$ & $\begin{array}{l}\text { 5'-CATAAAGGGCCCACTTGCTA-3' } \\
\text { 5'-AGCTGGGCCAATATCTCCTT-3' }\end{array}$ & 40 \\
\hline Nestin & $\begin{array}{l}\text { Forward } \\
\text { Reverse }\end{array}$ & $\begin{array}{l}\text { 5'-AGGTTTGAAGACGCAGAGGA-3' } \\
\text { 5'-TTCGAGAGATTCGAGGGAGA-3' }\end{array}$ & 25 \\
\hline Brachyury & $\begin{array}{l}\text { Forward } \\
\text { Reverse }\end{array}$ & $\begin{array}{l}\text { 5'-GTGACTGCCTACCAGAATGA-3' } \\
\text { 5'-ATTGTCCGCATAGGTTGGAG-3' }\end{array}$ & 25 \\
\hline AFP & $\begin{array}{l}\text { Forward } \\
\text { Reverse }\end{array}$ & $\begin{array}{l}\text { 5'-AACTCTGGCGATGGGTGTT-3' } \\
\text { 5'-AAACTGGAAGGGTGGGACA-3' }\end{array}$ & 35 \\
\hline Stella & $\begin{array}{l}\text { Forward } \\
\text { Reverse }\end{array}$ & $\begin{array}{l}\text { 5'-AAGAGAAGGGTCCGCACTTT-3' } \\
\text { 5'-GATTTCCCAGCACCAGAAAA-3' }\end{array}$ & 25 \\
\hline Osteocalcin & $\begin{array}{l}\text { Forward } \\
\text { Reverse }\end{array}$ & $\begin{array}{l}\text { 5'-TCTCTCTGCTCACTCTGCTGG-3' } \\
\text { 5'-ACCGTAGATGCGTTTGTAGGC-3' }\end{array}$ & 35 \\
\hline aP2 & $\begin{array}{l}\text { Forward } \\
\text { Reverse }\end{array}$ & $\begin{array}{l}\text { 5'-ATGTGTGATGCCTTTGTGGGA-3' } \\
\text { 5'-TGCCCTTTCATAAACTCTTGT-3' }\end{array}$ & 35 \\
\hline$\alpha$-actin & $\begin{array}{l}\text { Forward } \\
\text { Reverse }\end{array}$ & $\begin{array}{l}\text { 5'-GGCATCCACGAAACCACCTA-3' } \\
\text { 5'-CACGAGTAACAAATCAAAGC-3' }\end{array}$ & 25 \\
\hline Flk1 & $\begin{array}{l}\text { Forward } \\
\text { Reverse }\end{array}$ & $\begin{array}{l}\text { 5'-CACCTGGCACTCTCCACCTTC-3' } \\
\text { 5'-GATTTCATCCCACTACCGAAAG-3' }\end{array}$ & 35 \\
\hline$\beta$-actin & $\begin{array}{l}\text { Forward } \\
\text { Reverse }\end{array}$ & $\begin{array}{l}\text { 5'-GGCCCAGAGCAAGAGAGGTATCC-3' } \\
5^{\prime} \text {-ACGCACGATTTCCCTCTCAGC-3' }\end{array}$ & 25 \\
\hline
\end{tabular}

containing the same base: DMEM, $1 \%$ NEAA, Pen/Strep and $\beta$ ME. To the base medium, the following factors were added: (i) ITS-CS: $1 \%$ ITS (Invitrogen), $1 \% \mathrm{FBS}$, $10 \mathrm{ng} / \mathrm{ml}$ TGF- $\beta 1$ (PeproTech), $10 \mathrm{ng} / \mathrm{ml}$ BMP-2 (PeproTech), $50 \mu \mathrm{g} / \mathrm{ml}$ ascorbic acid (Sigma), (ii) ITS-basic: 1\% ITS (Invitrogen), 1\% FBS and (iii) FBS-CS: $15 \%$ FBS, $1 \mu \mathrm{g} / \mathrm{ml}$ insulin (Sigma), $10 \mathrm{ng} / \mathrm{ml} \mathrm{TGF-} \beta 1,10 \mathrm{ng} / \mathrm{ml} \mathrm{BMP-2}$ and $50 \mu \mathrm{g} / \mathrm{ml}$ ascorbic acid. Medium was changed every day.

Cell viability. Cell viability was measured using the Vi-Cell XR (Beckman Coulter). Live/death cell number, viability and size were analyzed during differentiation in the different culture conditions. Only cells that were $2 \mu \mathrm{m}$ or larger were sorted for analysis.

Alcian Blue staining. Proteoglycans secreted by chondrocyte-derived ESCs were stained with Alcian Blue. Cells were fixed in $4 \%$ paraformaldehyde for $15 \mathrm{~min}$ and washed twice with PBS-T (0.05\% Tween-20 in PBS). They were then stained with Alcian Blue (1\%, pH 1.0) for $30 \mathrm{~min}$, followed by de-staining for $3 \mathrm{~min}$ in $0.1 \mathrm{~N}$ $\mathrm{HCl}$, and washed twice with PBS-T before visualization.

GAG assay. To measure total GAG accumulation during differentiation in the different culture conditions, cultures including floating cells and aggregates were digested with $100 \mu \mathrm{l}$ of papain digestion buffer $(4.5 \mathrm{U} / \mathrm{ml}$ in $50 \mathrm{mM}$ phosphate buffer $\mathrm{pH} 6.5$, with $2 \mathrm{mM} \mathrm{N}$-acetylcysteine and $2 \mathrm{mM} \mathrm{Na}_{2} \mathrm{EDTA}$; Sigma) for $4 \mathrm{~h}$ at $65^{\circ} \mathrm{C}$. Values are calculated from a standard with chondroitin sulfate-A sodium salt (Sigma), which was measured along with the samples at $510 \mathrm{~nm}$ in a Benchmark Plus microplate spectrophotometer (Bio-Rad). The readings were then normalized to dsDNA content, which was measured using a Bio-Photometer (Eppendorf).
Calcium assay. To measure calcium accumulation during differentiation in the different culture conditions, cultures including floating cells and aggregates were digested with $100 \mu \mathrm{l}$ of $10 \%$ formic acid. Values were calculated using the Arsenazo III (DCL), which was measured along with the samples at $650 \mathrm{~nm}$ in a Benchmark Plus microplate spectrophotometer. The readings were then normalized to dsDNA content, which was measured using a Bio-Photometer.

RNA isolation, RT-PCR. Total RNA was isolated from cell culture at 2, 4 and 6 days of differentiation using a RNeasy Mini kit (Qiagen) according to the manufacturer's instructions with on-column DNase I digestion. ${ }^{32}$ The amount of total RNA was measured using a Bio-Photometer. Here, $1 \mu \mathrm{g}$ of total RNA was used as a template for cDNA synthesis with Super Script III First-Strand Synthesis System (Invitrogen). The PCR reactions ( $20 \mu \mathrm{l}$ ) were carried out using Taq DNA Polymerase (Invitrogen) according to the manufacturer's instructions. PCR conditions were 3 min at $94^{\circ} \mathrm{C}, 30 \mathrm{~s}$ denaturation at $94^{\circ} \mathrm{C}, 45 \mathrm{~s}$ annealing at $55^{\circ} \mathrm{C}$ and $1 \mathrm{~min} 30 \mathrm{~s}$ extension at $72{ }^{\circ} \mathrm{C}$. Primers were designed based on the mouse sequence and BLASTed for their specificity at the National Center for Biotechnology Information (NCBI). Primer sequences and cycle number are described in Table 1. Amplified PCR products were analyzed on a $1.5 \%$ agarose gel. Imaging and scanning were performed using VWR M-20E ultraviolet light source and photographed by Kodak MI Image system.

Immunofluorescence. Cells were fixed in $4 \%$ paraformaldehyde for $15 \mathrm{~min}$ and washed twice with PBS-T. After permeabilization with PBS containing $0.1 \%$ Triton-X, cells were washed twice with PBS-T, blocked with PBS containing $3 \%$ BSA for $1 \mathrm{~h}$ at room temperature, and incubated with primary antibody (Santa Cruz; $1: 200)$ overnight at $4{ }^{\circ} \mathrm{C}$. Then cells were washed three times with PBS-T, incubated with Alexa Flour 488-conjugated secondary antibody (1:500) and DAPI (1:500) for 
$2 \mathrm{~h}$ at room temperature. ${ }^{33}$ Unbound secondary antibodies were removed by three washes with PBS-T. Fluorescent images were captured using a fluorescent microscope (IX70; Olympus, Japan) equipped with CCD camera (RT Color, Diagnostic, Spot Software V4.0.9).

In vivo cell implant assay. To determine the chondrogenic ability of cultured cells to differentiate in vivo, $4.5 \times 10^{5}$ cells dissociated by $0.1 \%$ trypsin-EDTA were injected into the muscle of SCID mice, using a 27-gauge syringe. SCID mice were obtained from Taconic and housed in the single-barrier animal facility of the Faculty of Medicine, University of Calgary ${ }^{34}$ After 4 weeks, animals were killed and tissue at the point of injection was dissected. These specimens were fixed in $4 \%$ paraformaldehyde and decalcified in $10 \%$ EDTA (pH 8.0) solution. After dehydration in ascending concentrations of ethanol and xylene, the specimens were embedded in paraffin. The paraffin sections were then deparaffinized, hydrated and stained with hematoxylin and eosin (H\&E), Alcian Blue and Alizarin Red $\mathrm{S}$ according to standard procedures.

TUNEL staining. To identify the apoptotic cells, TUNEL staining was performed with the in situ cell apoptosis detection kit according to the manufacturer's instructions (Roche). Briefly, the cells were fixed with $4 \%$ paraformaldehyde for $30 \mathrm{~min}$. Terminal deoxynucleotidyl transferase (TdT) and biotin-11-dUTP reactions were performed for $1 \mathrm{~h}$ at $37^{\circ} \mathrm{C}$. Fluorescent images were captured using a fluorescent microscope equipped with CCD camera.

Annexin V-FITC/PI. To determine the rate of apoptosis, Annexin V-FITC/PI labeling was performed with the apoptosis detection kit I according to the manufacturer's instructions (BD Pharmingen). Briefly, the cells dissociated by $0.1 \%$ trypsin-EDTA were washed once with ice-cold PBS and resuspended in binding buffer at a concentration of $1.0 \times 10^{6} \mathrm{cell} / \mathrm{s} / \mathrm{ml}$. Here, $5 \mu$ l each of Annexin V-FITC and PI was added and incubated for $15 \mathrm{~min}$ in the dark prior to a further addition of $400 \mu$ l PBS. Quantitative analysis of apoptotic level was performed using a flow cytometer (Becton Dickinson, Franklin Lakes, NJ, USA). In total, 10000 events were registered per sample with a FACS Calibur instrument and CellQuest software. ${ }^{29}$ Early apoptosis were sorted by Annexin V-FITC positive and PI negative. Late apoptosis were sorted by Annexin V-FITC and PI positive.

Statistical analysis. Means \pm S.E.M. were calculated and statistically significant differences between two groups were determined using the Student's $t$-test at $P<0.05$ (Excel, Microsoft).

Acknowledgements. We thank Drs John R Matyas and Michael S Kallos for providing scientific advice and support for this study. We also thank Rosaline Alfred and Katherine Chiang for assistance with the FACScan and Vi-Cell XR analysis. This study was supported by National Institutes of Health RO1 grant AR053738-01. AY is an Alberta Heritage Foundation of Medical Research Postdoctoral Fellow (2007) and Japan Society for the Promotion of Science Postdoctoral Fellow for Research Abroad (2008). DER is an Alberta Heritage Foundation of Medical Research Senior Scholar.

1. Evans MJ, Kaufman MH. Establishment in culture of pluripotential cells from mouse embryos. Nature 1981; 292: 154-156.

2. Martin GR. Isolation of a pluripotent cell line from early mouse embryos cultured in medium conditioned by teretocarcinoma stem cells. Proc Natl Acad Sci USA 1981; 78: 7634-7638.

3. Itskovitz-Eldor J, Schuldiner M, Karsenti D, Eden A, Yanuka O, Amit M et al. Differentiation of human embryonic stem cells into embryoid bodies compromising the three embryonic germ layers. Mol Med 2000; 6: 88-95.

4. Keller GM. In-vitro differentiation of embryonic stem cells. Curr Opin Cell Biol 1995; 7: 862-869.

5. Metallo CM, Azarin SM, Ji L, de Pablo JJ, Palecek SP. Engineering tissue from human embryonic stem cells. J Cell Mol Med 2008; 3: 709-729.

6. Yamada T, Yoshikawa M, Kanda S, Kato Y, Nakajima Y, Ishizaka S et al. In-vitro differentiation of embryonic stem cells into hepatocyte-like cells identified by cellular uptake of indocyanine green. Stem Cells 2002; 20: 146-154.

7. Shimko DA, Burks CA, Dee KC, Nauman EA. Comparison of in-vitro mineralization by murine embryonic and adult stem cells cultured in an osteogenic medium. Tissue Eng 2004; 10: 1386-1398.
8. Karp JM, Ferreira LS, Khademhosseini A, Kwon AH, Yeh J, Langer RS. Cultivation of human embryonic stem cells without the embryoid body step enhances osteogenesis in-vitro. Stem Cells 2006; 24: 835-843.

9. Duplomb L, Dagouassat M, Jourdon P, Heymann D. Differentiation of osteoblasts from mouse embryonic stem cells without generation of embryoid body. In-vitro Cell Dev Biol Anim 2007; 43: 21-24.

10. Cukierman E, Pankov R, Stevens DR, Yamada KM. Taking cell-matrix adhesions to the third dimension. Science 2001; 294: 1708-1712.

11. Schmeichel KL, Bissell MJ. Modeling tissue-specific signaling and organ function in three dimensions. J Cell Sci 2003; 116: 2377-2388.

12. Mackay AM, Beck SC, Murphy JM, Barry FP, Chichester CO, Pittenger MF. Chondrogenic differentiation of cultured human mesenchymal stem cells from marrow. Tissue Eng 1998; 4: 415-428.

13. Tanaka TS, Kunath T, Kimber WL, Jaradat SA, Stagg CA, Usuda M et al. Gene expression profiling of embryo-derived stem cells reveals candidate genes associated with pluripotency and lineage specificity. Genome Res 2002; 12: 1921-1928.

14. Egusa H, Schweizer FE, Wang CC, Matsuka Y, Nishimura I. Neuronal differentiation of bone marrow-derived stromal stem cells involves suppression of discordant phenotypes through transcriptional regulation. J Biol Chem 2005; 280: 23691-23697.

15. Egusa $\mathrm{H}$, lida K, Kobayashi M, Lin TY, Zhu M, Zuk PA et al. Downregulation of extracellular matrix-related gene clusters during osteogenic differentiation of human bone marrow- and adipose tissue-derived stromal cells. Tissue Eng 2007; 13: 2589-2600.

16. zur Nieden NI, Kempka G, Rancourt DE, Ahr HJ. Induction of chondro-, osteo- and adipogenesis in embryonic stem cells by bone morphogenetic protein-2: effect of cofactors on differentiating lineages. BMC Dev Biol 2005; 5: 1.

17. Hwang YS, Polak JM, Mantalaris A. In vitro direct chondrogenesis of murine embryonic stem cells by bypassing embryoid body formation. Stem Cells Dev (in press).

18. Vormann J, Förster C, Zippel U, Lozo E, Günther T, Merker H et al. Effects of magnesium deficiency on magnesium and calcium content in bone and cartilage in developing rats in correlation to chondrotoxicity. Calcif Tissue Int 1997; 61: 230-238.

19. Nakayama N, Duryea D, Manoukian R, Chow G, Han CY. Macroscopic cartilage formation with embryonic stem-cell-derived mesodermal progenitor cells. J Cell Sci 2003; 116: 2015-2028.

20. Sugiki T, Uyama T, Toyoda M, Morioka H, Kume S, Miyado $\mathrm{K}$ et al. Hyaline cartilage formation and enchondral ossification modeled with KUM5 and OP9 chondroblasts. $\mathrm{J}$ Cell Biochem 2007; 100: 1240-1254

21. Hegert C, Kramer J, Hargus G, Müller J, Guan K, Wobus AM et al. Differentiation plasticity of chondrocytes derived from mouse embryonic stem cells. J Cell Sci 2002; 115: 4617-4628.

22. Kramer J, Hegert C, Guan K, Wobus AM, Müller PK, Rohwedel J. Embryonic stem cell-derived chondrogenic differentiation in-vitro: activation by BMP-2 and BMP-4. Mech Dev 2000; 92: 193-205.

23. Jukes JM, Moroni L, van Blitterswijk CA, de Boer J. Critical steps toward a tissue-engineered cartilage implant using embryonic stem cells. Tissue Eng Part A 2008; 14: 135-147.

24. Jukes JM, Both SK, Leusink A, Sterk LM, van Blitterswijk CA, de Boer J. Endochondral bone tissue engineering using embryonic stem cells. Proc Natl Acad Sci USA 2008; 105 6840-6845.

25. Toh WS, Yang Z, Liu H, Heng BC, Lee EH, Cao T. Effects of culture conditions and bone morphogenetic protein 2 on extent of chondrogenesis from human embryonic stem cells. Stem Cells 2007; 25: 950-960.

26. Hessler JA, Budor A, Putchakayala K, Mecke A, Rieger D, Banaszak Holl MM et al. Atomic force microscopy study of early morphological changes during apoptosis. Langmuir 2005; 21: $9280-9286$

27. Hirsch MS, Cook SC, Killiany R, Hartford Svoboda KK. Increased cell diameter precedes chondrocyte terminal differentiation, whereas cell-matrix attachment complex proteins appear constant. Anat Rec 1996; 244: 284-296.

28. Doetschman TC, Eistetter H, Katz M, Schmidt W, Kemler R. The in vitro development of blastocyst-derived embryonic stem cell lines: formation of visceral yolk sac, blood islands and myocardium. J Embryol Exp Morphol 1985; 87: 27-45.

29. Smith AG. Culture and differentiation of embryonic stem cells. J Tissue Culture Methods 1991; 13: 89-94.

30. Yamashita A, Takada T, Nemoto K, Yamamoto G, Torii R. Transient suppression of PPARgamma directed ES cells into an osteoblastic lineage. FEBS Lett 2006; 580: 4121-4125.

31. Cormier JT, zur Nieden NI, Rancourt DE, Kallos MS. Expansion of undifferentiated murin embryonic stem cells as aggregates in suspension culture bioreactors. Tissue Eng 2006; 12: $3233-3245$

32. Yamashita A, Takada T, Omatsu-Kanbe M, Nemoto K, Matsuura H, Yamamoto G et al. Monkey embryonic stem cells differentiate into adipocytes in-vitro. Cloning Stem Cells 2006; 8: 3-9

33. Yamashita A, Takada T, Narita J, Yamamoto G, Torii R. Osteoblastic differentiation of monkey embryonic stem cells in-vitro. Cloning Stem Cells 2005; 7: 232-237.

34. zur Nieden NI, Cormier JT, Rancourt DE, Kallos MS. Embryonic stem cells remain highly pluripotent following long term expansion as aggregates in suspension bioreactors. J Biotechnol 2007; 129: 421-432. 